\title{
La Falacia sobre la Severidad de las Penas en el Derecho criminal
}

Fallacy on the Severity of Penalties in Criminal Law

\author{
Dra. Iris Edith La Salvia, PhD (c) \\ Relatora de la Suprema Corte de Justicia de la Prov. de Buenos Aires
}

Artículo Original (Investigación)

RFJ, No. 2, 2017, pp. 67-78, ISSN 2588-0837

\begin{abstract}
RESUMEN: el siguiente análisis responde a la necesidad de reflexión que se impone en el ámbito del Derecho Penal en Argentina, a fin de establecer las múltiples causas que desembocaron en los actuales índices de criminalidad, que progresivamente han instalado en la sociedad argentina el temor habitual de convertirse en víctima de delito -en particular violentos-. Asimismo, se analiza hasta qué punto es errada la opinión pública que atribuye dicha situación a una supuesta flexibilización de la legislación penal al tiempo que reclama mayor severidad penal, indicándose finalmente aquí las múltiples causas y los enfoques que aporten posible solución al Estado de cosas actual. Las falacias constituyen razonamientos seductores, aúnque erróneos, resultan psicológicamente persuasivos y por ello se convierten en peligrosas trampas a la razón. El intelecto debe imponerse a ellas, apartándose en este caso de la "falacia de la causa falsa", para profundizar en las variables relevantes y las causas eficientes de la situación.
\end{abstract}

PALABRAS CLAVES: índice de criminalidad, inseguridad, reformas en legislación penal, agravamiento de penas y figuras penales, corrupción.

ABSTRACT: the following analysis responds to the need of reflex ion imposed in the criminal law area for argentine judicial system, lawyers and operators, in order to establish the multiple causes that generate the present criminal index, which have been installing fear, in the argentine society, to become a victim of a crime, particularly violent. It also discusses how much is misjudged the public opinion that attaches the situation to the penal law relaxation, indicating finally the multiple causes and approaches that give solution to the present state of things. 
KEYWORDS: criminality index, insecurity, penal legislation reforms, penalty aggravation and legal figures, corruption.

\section{INTRODUCCIÓN}

La afición a los autores clásicos me devolvió cierto día a las páginas que Francesco Carrara escribió en su Programma del corso di diritto criminale al abordar las cuestiones vinculadas a la pena. La inquietante concordancia de sus previsiones con la realidad que experimenta la sociedad argentina actualmente en el ámbito criminal me llevó entonces a estas reflexiones.

En primer término, debo recordar que en la obra citada - más precisamente en el parágrafo 616 del volumen segundo- el autor destaca que dentro del contexto social en el que se comete, el delito ofende materialmente a una o varias personas, pero agravia a la sociedad al violar sus leyes y ofende moralmente a todos los ciudadanos al disminuir en ellos el sentimiento de la propia seguridad y crear el peligro del mal ejemplo. Con su comisión nace el peligro que amenaza a todos los ciudadanos; que el delincuente si permanece impune renueve contra otros sus ofensas y que otros, incitados por el mal ejemplo, se entreguen también a violar las leyes. La pena debe reparar este daño moral mediante el restablecimiento del orden que se ha visto conmovido por el desorden del delito, entonces todos los ciudadanos que temían nuevas ofensas, dejan de temerlas. De allí deduce la necesidad de efectividad de la pena, para que los ciudadanos no tengan razón de temer que el delincuente, liberado demasiado pronto, reincida en sus ofensas. Cuando el delito pasa del Estado de previsión al Estado de realidad, la fuerza moral de la amenaza quedaría destruida si la pena no se convirtiera también en una realidad. Solo a partir de ese momento ella se completa.

Hasta aquí, en términos generales el pensamiento de Carrara. Más allá del estilo y terminología propios de su época, el esquema permite apreciar los aspectos relevantes de la cuestión, con la claridad propia y encomiable que siempre tienen los clásicos. En ese punto de la relectura me preguntaba cuál sería el motivo o las múltiples circunstancias que en la sociedad argentina actualmente han llevado a la opinión pública, los medios de comunicación y hasta ciertos discursos políticos a invocar en sus descripciones ese sentimiento de temor en los honrados e impunidad en los delincuentes tan bien definido por Carrara. 
Inquietud actualizada ante el cumplimiento de sus predicciones sobre las consecuencias lamentables del desequilibrio, en particular la recurrencia de incidentes por venganza privada o castigos por mano propia verificados en distintas oportunidades. Emprendí entonces un análisis de la situación desde el punto de vista jurídico, a fin de establecer en que intersección de los complejos ámbitos implicados (jurídico, sociológico, cultural y económico) surgió el Estado de cosas referido, que se encuentra a la vista de cualquier observador diligente.

\section{CRIMINALIDAD Y CONTROL DEL ESTADO}

En principio debe aclararse que el Estado óptimo de la sociedad -donde las fuerzas del modelo de Carrara se mantienen en equilibriono es una situación utópica en la que no existe delito, sino aquella donde la criminalidad se mantiene en la frontera y se coloca bajo el control del Estado, porque los hechos punibles cometidos son aclarados en alto porcentaje y castigados sin desconsideración hacia la persona involucrada - conf. Jescheck- pues señala este último autor, tan pronto como el Derecho Penal no pueda garantizar más la seguridad y el orden -con las garantías propias del Estado de Derecho- existe el peligro que los ciudadanos se tomen la justicia por su mano y que los fuertes se impongan a los débiles sin consideración alguna.

Solo para mencionar inicialmente el aspecto más tangible de la cuestión -la punición del delito- se puede advertir que las condiciones de eficacia que los clásicos exigían de la pena, sintetizadas en su carácter ejemplar, aflictivo y fundamentalmente en su certeza legal y celeridad, no han perdido vigencia en nuestros días. Precisamente una de las características más destacadas junto a la ejemplaridad es la certeza legal de su aplicación, más decisiva que su severidad. Ello es demostrativo de la ineficacia del abordaje simplista sobre la severidad de las penas, en tanto implica desconocer las diferencias entre las diversas formas de criminalidad -crimen organizado y delincuencia común- que requieren respuestas diferentes, al tiempo que ignora los problemas estructurales de las fuerzas de seguridad y la situación de alta vulnerabilidad en que se encuentran amplios sectores de la sociedad, con exclusión laboral y educativa. Asimismo, en cuanto a la celeridad, la pena debe ser aplicada en tiempo y forma por la autoridad pública a fin de disuadir la venganza por mano propia, al respetar las garantías y dignidad del condenado. No obstante, lo expresado, debo 
resaltar que la pena, aúnque el más visible, constituye solo uno de los múltiples aspectos involucrados en esta cuestión.

En segundo lugar, debo advertir que ante la multiplicidad de factores psicológicos, sociales, económicos, culturales y ambientales que tienen incidencia sobre el tema, decidí relevar exclusivamente aquellos pertenecientes al ámbito del Derecho Penal y los directamente vinculados al mismo, por resultar en cierto modo el más representativo de los aspectos de la temática y el único al alcance de mi especialidad. De ello surgirán, consecuentemente, las fortalezas y debilidades del examen que aquí propongo.

\section{TRES PILARES FUNDAMENTALES}

Ante la necesidad de establecer dentro del campo del Derecho Penal cuáles podrían haber sido los factores relevantes que conformaron la situación actual decidí dirigir el enfoque hacia tres pilares fundamentales que inciden en la problemática descrita:

1. La legislación -al analizar las reformas normativas del ordenamiento penal argentino (y eventualmente procesal penal) desde el año 1984 hasta principios de 2015- a fin de establecer su tendencia.

2. El contexto social y la estadística en relación a la comisión de delitos, al considerar en ello a las víctimas.

3. La aplicación de la ley y los resultados estadísticos sobre delitos esclarecidos, personas detenidas y condenadas.

Todo ello en cuanto al ordenamiento jurídico y a la sociedad argentina en el lapso ya referido, estructurándolo en tres períodos; desde el año 1984 al 2000, para continuar desde este hasta 2010 y finalmente evaluar lo que va de la presente década hasta los primeros meses del año 2015.

\subsection{La legislación nacional: Las modificaciones legislativas del Código Penal Argentino}

Los períodos analizados corresponden a etapas en las cuales el Estado de Derecho ya se encontraba vigente, al tiempo que coinciden con 
el inicio del siglo XXI al ofrecer una visión dinámica de la legislación penal hasta la actualidad.

La primera hipótesis, descripta en la sección anterior acerca de la opinión pública y la expresión de algunas autoridades, vincula una supuesta flexibilización o acentuada benignidad en las leyes penales sustantivas -y de algunos institutos procesales de incidencia- dictadas desde 1984 hasta 2015 con el Estado actual de la cuestión criminal en la sociedad argentina, para concluir que esa pérdida de severidad sería su causa eficiente.

Para verificarla se debe conocer someramente el contenido de las modificaciones introducidas en el código penal en ese período, a fin de establecer si ellas han incorporado mayor o menor rigor en los institutos y figuras penales afectadas. A tal fin, al partir de la redacción de los artículos del Código Penal Argentino vigente en el año 1984 evalué comparativamente el carácter de las reformas introducidas en el período 2000/2010 y seguidamente las que operaron en el período 2010/2015, lo que puede cotejarse en el anexo -al que me remito-.

El detalle que surge al relevar el contenido de las reformas más importantes verificadas en los períodos en estudio exime de cualquier comentario por ser evidente que en términos globales aquellas introdujeron mayor rigor en las reglas aplicables, al destacar la incorporación de figuras agravadas o el aumento de penalidades en las existentes. Algo similar sucedió con los institutos procesales. En consecuencia, el Estado de cosas descripto al comienzo no puede obedecer a la mayor benignidad o flexibilización de la legislación penal -pese a la creencia más popular en la sociedad argentina- en tanto se ha verificado que las modificaciones introducidas agravaron en su mayoría penas y figuras, $\mathrm{y}$ en ocasiones introdujeron supuestos antes no sancionados o figuras específicas no contempladas con anterioridad. Cabe entonces relevar los restantes parámetros.

\subsection{Análisis estadístico y contexto social}

En Argentina, particularmente durante las décadas de los ochenta y noventa se registró un aumento sostenido de los índices de delito, duplicándose las agresiones contra la propiedad y elevándose muy por encima de la media histórica la tasa de homicidios dolosos -aúnque inferior a la de otros países de la región- (Ciafardini, 2006) al ser tam- 
bién un dato de interés señalado por este autor el cambio -registrado a mediados de los años 90- en la edad promedio de los autores de delitos violentos contra la propiedad, al aumentar la participación de jóvenes entre 18 y 25 años en el robo con armas y al aparecer en escena los menores de 17 años, dentro de un deterioro social con "complejos procesos de degradación de las formas de organización económica y social, que incluyen aspectos individuales, familiares, institucionales y políticos”. En ese panorama se insertó también un factor que no había tenido la misma incidencia en etapas anteriores y que aquí debo destacar, la droga y su influencia en la comisión de delitos más violentos, al obrar a la vez como detonante o agravante de patologías mentales, con la consiguiente incertidumbre tanto en las reacciones del delincuente al momento de cometer el hecho, cuanto en la evolución de su eventual patología, que ya no resultaría predecible ante la incorporación de dicha variable, al dificultar su abordaje y tratamiento, al complicar así también el campo de la salud mental además del criminológico. La década del 90 presentó el desarrollo de políticas de flexibilización laboral, crecimiento del desempleo y consecuente aumento de la marginalidad, al tiempo que -como señala Ciafardini- continuaban las consecuencias deletéreas de la última dictadura militar que en su momento había disuelto completamente las instancias institucionales legítimas, así como el tejido sociopolítico, lo cual posibilitó el surgimiento de sistemas de asociación ilícita entre funcionarios, políticos e integrantes de fuerzas de seguridad. En ese complejo contexto, inserto a su vez dentro de la grave situación que viene al experimentar toda la región latinoamericana en la evolución de la tasa de criminalidad, se fue al desarrollar un perfil diferente en el crimen urbano y con él de la propia sociedad -que llega hasta nuestros días- atento el costo y distorsión de los parámetros normales producidos por la pérdida de vidas, las pérdidas materiales, la distorsión de presupuestos estatales y privados en la adopción de medidas extraordinarias de seguridad y la afectación de la salud mental de la población, derivada de vivir permanentemente al tomar precauciones y con miedo a convertirse en víctima de delito.

Como señalan los especialistas se considera aceptable en una sociedad moderna el guarismo de 5 homicidios cada 100.000 habitantes al año, al ser alarmante entre 5 y 8 . Téngase en cuenta que en el año 2011 la tasa de homicidios dolosos cada 100.000 habitantes en la provincia de Buenos Aires que es una de las que registra mayor índice de criminalidad en el país fue del 7,7 según datos oficiales de la Dirección Provincial de Política de Prevención del Delito en su "Estadística Cri- 
minal de la provincia de Buenos Aires 2011", en tanto en la estadística 2012 del sistema nacional de política criminal se indica un 5,5\% pero al no incluir la misma a la provincia de Buenos Aires se distorsiona considerablemente ese valor en la realidad.

A nivel nacional, la encuesta (EDSA) de la Universidad Católica de Argentina para el período 2004-2010 arrojó indicadores del grado de vulnerabilidad en que se encuentra la sociedad argentina ante la criminalidad, al registrar un crecimiento sistemático de los índices de delincuencia durante el citado período, al pasar del 21,7\% en 2004 al $30,9 \%$ en 2010 respecto de personas que fueron víctima de un delito. En el año 2012 fue del 30,3\%. Cabe señalar en este punto que desde la década del 60 se comenzó a utilizar en los países occidentales la búsqueda de información mediante encuestas que no fueran elaboradas a partir de registros oficiales a fin de disminuir los sesgos y la ignorancia sobre la "cifra negra" del delito, por ello resulta de especial relevancia la información obtenida por Universidades e Institutos en forma independiente. Dichas estadísticas e informes también demuestran el aumento de los recursos privados como vigilancia policial (que aumentó del $36,2 \%$ en 2005 al 50,4\% en 2010) y la relación existente entre vivir en un barrio donde es habitual la venta o tráfico de drogas y el haber sufrido un hecho delictivo (del $27,7 \%$ cuando no hay tráfico de drogas en su barrio al 35,2\% cuando lo hay durante el año 2012).

Una investigación relevante por la especificidad de su estudio, en tanto constituye un sector de la población muy expuesto al delito, fue realizada por los investigadores de la Universidad Nacional de La Plata, Facultad de Ciencias Médicas (Folino, et al 2012), en torno a la victimización de comerciantes en la ciudad de La Plata entre octubre 2011 a septiembre de 2012. Los resultados indicaron una tasa de victimización para el año previo a la entrevista del 19.9\%, al haber sufrido el $48 \%$ de las víctimas más de un delito en ese período. El $21 \%$ de los hechos correspondió al delito de hurto, el $71 \%$ a robo el $8 \%$ restante daño o estafa. En el $75 \%$ de los casos el autor se fugó con algún valor. 15 víctimas resistieron al delito; 5 quedaron levemente heridas, 1 gravemente y una de las víctimas informó haber sido abusada sexualmente durante el robo al comercio. La conducta de los autores de delitos evidenció desestimación de los riesgos de ser identificados o de actuar en zonas y horarios altamente poblados, al ocurrir algunos hechos en proximidades de Comisarías y el $62,7 \%$ de ellos entre las 14 y las $00.00 \mathrm{hs}$, al prevalecer la comisión en horas de la tarde en zonas de alta densidad y en la noche en las de baja densidad. La edad promedio 
de los autores fue de 25 años al ser el mínimo de 9 años, utilizándose arma de fuego en el $77 \%$; arma blanca $20 \%$, algún otro objeto usado como arma impropia el 3\%. El 57\% de las víctimas hizo la denuncia en Comisaría, $1 \%$ en Fiscalía y el $42 \%$ no hizo denuncia (obsérvese aquí el interés de las encuestas no oficiales en tanto recogen la cifra negra del delito que pasa absolutamente inadvertida para los registros oficiales). Las mujeres tuvieron casi el doble de victimización. Una proporción relevante de víctimas expresó la necesidad de haber recibido apoyo de algún organismo especializado, lo que no sucedió. Se advirtió también en dicha investigación la proliferación de locales con notables medidas de seguridad como vidrios blindados (incluso en farmacias y heladerías). Se destacó que los autores de los delitos no se preocupaban por ser reconocidos, manejándose con impunidad. Este es el Estado de cosas que se verifica en la sociedad argentina actual, reflejado sin mayor hesitación por las investigaciones e informes especializados.

\subsection{La articulación entre el contexto y la normativa legal: aplicación de la Ley}

Otra faceta de incidencia en el tema estudiado es la referida a la investigación judicial y a la forma en que los tribunales aplican la ley, ello si acaso se revelara una tendencia indulgente o se advirtiera cierta ineficacia en los mecanismos de investigación de los delitos cometidos y efectivamente denunciados, con efectos similares a los atribuidos a la flexibilización normativa. Para verificarlo -más allá de las reservas que puede merecer en sí el método- es ilustrativa la estadística criminal del año 2011 correspondiente a la provincia de Bs. As; al 1 de marzo de 2012 se registraron en materia de homicidios el 74\% esclarecidos con imputado detenido; con imputado identificado, pero aún no habido $9 \%$ y no esclarecidos el $17 \%$. La franja etaria de las víctimas entre los 19 y los 40 constituye un 5,5\% de homicidios verificados en niños entre 0 y 15 años de edad de los cuales el 75\% se debió a conflictos familiares. En cuanto a lugares de comisión del homicidio el $32 \%$ fue en vía pública, $29 \%$ sin datos, $23 \%$ en domicilio particular, $6 \%$ en comercio, $3 \%$ en rodados, $2 \%$ en comisaría o cárcel, $5 \%$ en otro lugar. Respecto de las violaciones el $49 \%$ sucedió en el ámbito intrafamiliar, especialmente de víctimas menores, al existir en el $73 \%$ de los casos conocimiento previo entre víctima y autor y ningún conocimiento en el $27 \%$. En cuanto a los robos el $54,61 \%$ fue en la vía pública, el $24,57 \%$ en domicilio particular, el $11,61 \%$ en comercio, $2,81 \%$ en rodados, otro lugar $4,55 \%$, cárcel $0,02 \%$ sin datos $1,84 \%$. En las causas de nar- 
cotráfico la estadística oficial indicó un notable incremento, del $28 \%$ mayor en relación al año 2009.

Respecto de las investigaciones penales en el ámbito de la justicia nacional, según el sistema nacional de estadísticas judiciales SNEJ tomadas del Registro Nacional de Reincidencia y Estadística Criminal en 2012 se dictaron 29.773 sentencias condenatorias; 4979 por delitos contra las personas al ser 1463 por homicidios dolosos, 15.521 por robos, 2872 por delitos contra la seguridad pública, 2542 por delitos contra la administración pública, 2372 por infracciones a la ley de estupefacientes 23737, y 1836 contra la integridad sexual, entre los más representativos. En cuanto a las penas privativas de libertad impuestas 18.084 casos fueron hasta 3 años, entre 3 y 5 años se aplicaron en 5864 casos y más de 5 años 5458, aplicándose penas perpetuas en 104 casos, las inhabilitaciones especiales 4579 casos, las multas 1857 casos. Debe destacarse en este informe la ausencia de datos respecto de varias provincias de gran incidencia en la tasa de criminalidad, así como la ausencia de datos sobre el total de causas en trámite en todas las provincias.

En lo que atañe al contenido en la actuación de los tribunales, interesa relevar criterios jurisprudenciales de interpretación aplicados en materias representativas, para conocer la mayor o menor severidad de los mismos. Esto releva someramente que algunos de ellos -ante la imposibilidad de hacerlo exhaustivamente- referidos a temas de incidencia en fallos rectores encontramos, entre otros; que la CSJN admite el distinto tratamiento dado por la ley a quienes en los términos del art. 50 del C.P., cometen un nuevo delito, respecto de quienes no lo han cometido, fundado en el desprecio hacia la pena por quien ya la ha experimentado CS “L Eveque, Ramón.”16/08/1988 La Ley online al tiempo que considera que el principio constitucional del non bis in ídem no impide al legislador tomar en cuenta la anterior condena -como dato formal y objetivo- para ajustar con mayor precisión el tratamiento penitenciario que considere adecuado para los supuestos en que el individuo incurriese en una nueva infracción criminal,. CS "Valdez, Enrique y otra" 21/04/1988 La Ley online y más recientemente en "Arévalo, Martín Salomón s/causa n 11.835" A. 558 XLVI del 27 de mayo de 2014 con expresa remisión a los precedentes "Gómez Dávalos" -Fallos 308:1938- "L’Eveque" -Fallos 311:145. En similar sentido se ha expedido la SCJBA respecto de la condena anterior en los términos del art. 50 del C.P., al igual que en relación con la agravante del art. 189 bis 2 inc. final "P. 114.455 Q. D, F. Recurso extraordinario 9/10/2013". En los homicidios agravados del art. 80 inc. $1^{\circ}$ la SCJBA 
ha delimitado claramente la aplicación del párrafo final referido a las circunstancias extraordinarias de atenuación que harían procedente la figura privilegiada entre otros en P. 104120 "A.V., recurso extraordinario 7/5/2014 y más recientemente en 2015 P. 116.768 "B.R". Por su parte, la CS estableció en "Villarruel, D" del 27/5/2004 -La Ley on line- la procedencia de la agravante del robo por el uso de armas de fuego aún cuando aquella no fue secuestrada, si la existencia y utilización pudo probarse por otros medios, tales como testimonios. En los casos en que no se pudo establecer la idoneidad del arma, teniéndose por acreditado su empleo, la SCJBA ha subsumido el hecho en el art. 166 inc. 2 apartado 3 ley 25.882 "J.JE. LLBA 2006-483 8/3/2006.

Otro dato relevante es la cantidad de detenidos, en tanto las pruebas refutan la supuesta impunidad o liberación inmediata que ligeramente se afirma en la opinión y los medios de comunicación. En octubre de 2014 se informó record histórico de personas detenidas en el SPF al llegar a 10.322 al haber aumentado en forma constante desde 2007 (14\%). En tanto la situación en la provincia de Bs.As., había alcanzado en mayo de 2014 la cifra más alta de los últimos quince años con 33.166 detenidos.

Como se aprecia, la actuación de la ley por parte de los tribunales no evidenció cambios drásticos hacia una flexibilización, al mantener en cambio la rigurosidad que las propias modificaciones legislativas fueron al delinear, al tiempo que se mantuvo en márgenes aceptables la cantidad de delitos investigados con autores imputados y condenados. De allí que deban descartarse las hipótesis simplistas que atribuyen el Estado de cosas descripto al comienzo como producto de la benignidad de las leyes penales o su actuación por los tribunales del país.

\section{CONCLUSIONES}

Es evidente que las hipótesis simplistas ligeramente afirmadas en la opinión del común, medios de comunicación y ciertos discursos políticos, al alegar la necesidad de mayor severidad en las leyes o actuación de los tribunales para revertir un Estado de cosas supuestamente originado en la benignidad legal o judicial contrastan con un análisis crítico de los datos y supuestos. La sociedad argentina padece aún los efectos deletéreos de la política que tuvo auge en los noventa-aúnque venía dándose desde la década del 70- al afectar negativamente a amplios sectores de la sociedad y al minar las fuentes de trabajo y formación, con altos niveles de desigualdad en acceso a la salud y educación, 
al tiempo que extendió la corrupción institucional y en las fuerzas del orden. Ninguna hipótesis simplista resultará operativa para modificar tal Estado de cosas, ya quienes provocaron esa degradación de la sociedad intentaron al mismo tiempo implementar las políticas de mano dura importadas de países desarrollados con circunstancias sociales diferentes a las locales, al desconocer así el verdadero origen de la delincuencia local y evidentemente fracasaron. Resulta claro que no puede darse la misma respuesta penal a la delincuencia organizada que a la común y la sociedad argentina actual presenta ambos tipos de criminalidad (bandas de narcotráfico, organizaciones de tráfico ilícito de medicamentos, de trata de personas, de robo y comercialización de automotores, entre otras, además de la delincuencia juvenil y la común). Nada más ineficiente que una respuesta única para especies tan diversas. El mero agravamiento de las penas y figuras o la reducción de los supuestos de excarcelación no han demostrado históricamente en ningún país reducción de los índices de criminalidad (Kliksberg 2008). Para ello deben removerse y eliminarse las causas estructurales que la provocan, lo que requiere una sostenida labor a través de políticas de formación individual y de distribución, desarrollo e integración económico-social de largo plazo adecuadas al contexto, pero exige también la decisión política de eliminar los factores y elementos mediante los cuales en distintos niveles opera la corrupción. De tal modo, al enaltecer las instituciones podrá devolverse la dignidad a quienes integran la sociedad argentina actual y deben gozar de sus Derechos personalísimos sin temor a ver segada su integridad o su existencia por la falta de previsión y las acciones desacertadas de sus mandatarios.

\section{REFERENCIAS BIBLIOGRÁFICAS}

Carrara, F. (1957). Programa de Derecho criminal Parte general. V.II. Bogotá. Temis.

CELS. (2014). Datos del RUD, Ministerio de Justicia y Seguridad de la provincia de Bs.As.

Ciafardini, M. (2006). Delito urbano en la Argentina. Bs.As. Ariel.

Encuesta de la Deuda Social. UCA 2004-2010. Recuperado de http:// www.uca.edu.ar 
Folino, J. et al. (2012). Victimización de comerciantes en la ciudad de La Plata. Revista Intercambios $\mathrm{N}^{\circ} 6$ UNLP. Recuperado en http:// intercambios.jursoc.unlp.edu.ar/

Jescheck, H. (2002). Tratado de Derecho Penal. Parte general Granada. Ed. Comares.

Kliksberg, B. (2008). ¿Cómo enfrentar la inseguridad en América Latina? Nueva Sociedad No 215 mayo-junio 2008 ISSN 0251-3552. Recuperado de http://. www.nuso.org

Procuración Penitenciaria de la Nación. Récord histórico de personas detenidas Recuperado de http:// www.ppn.gov.ar

Sistema Nacional de Estadísticas Judiciales SNEJ 2012. http://www. jus.gob.ar/areas-tematicas/estadisticas-de-politica-criminal/mapa. aspx

Recibido: $10 / 08 / 2017$

Aceptado: 21/11/2017

Dra. Iris Edith La Salvia, PhD (c): Relatora de la Suprema Corte de Justicia de la Provincia de Buenos Aires.

Correo electrónico: correoiris@gmail.com 